\title{
Dispersed Points and Geometric Embedding of Complete Bipartite Graphs
}

\author{
Hiroshi Maehara \\ College of Education, Ryukyu University, Okinawa, Japan
}

\begin{abstract}
The minimum dimension needed to represent $K(m, n)$ as a "unit neighborhood graph" in Euclidean space is considered. Some upper and lower bounds on this dimension are given, and the exact values of the dimension are calculated for $m \leq 3, n \leq 10$.
\end{abstract}

\section{Introduction}

Let $X$ be a nonempty subset of Euclidean $n$-space $E^{n}$. Then the graph having vertex set $X$ and edge set

$$
\{x y:|x-y| \leq 1, x, y \in X, x \neq y\}
$$

(where $\mid$ denotes the Euclidean norm) is called the unit neighborhood graph on $X$. For a (finite simple) graph $G$, the sphericity of $G$, sph $G$, is the minimum dimension $n$ such that $G$ is isomorphic to a unit neighborhood graph in $E^{n}$. Concerning sphericity and some other geometric graph dimensions, see, e.g., [1]-[4] and [6]-[12]. In this paper we give some bounds on the sphericity of the complete bipartite graph $K(m, n)$ and derive exact values of $\operatorname{sph} K(m, n), m \leq 3$, $n \leq 10$. Since sphericity is a hereditary property (that is, if $H$ is an induced subgraph of $G$, then sph $H \leq \operatorname{sph} G$ ), these results will be useful to estimate sph $G$ for other graphs $G$.

A nonempty subset $X$ of a Euclidean space is said to be dispersed if the unit neighborhood graph on $X$ is an edgeless graph, that is, $|x-y|>1$ for any two distinct points $x, y$ of $X$. The maximum cardinality of a dispersed subset of a set $Y$ is called the dispersed point number of $Y$ and denoted by dpn $Y$. For example, the dispersed point number of a unit circle is 5 . Let us denote by $S^{k}(r)$ a hypersphere of radius $r$ in $k$-space $E^{k}$, and put

$$
N(k, m)=\operatorname{dpn} S^{k}(s(m)),
$$

where $s(m)=\sqrt{(m+1) /(2 m)}$. 
Theorem 1. $\operatorname{sph} K(m, N(k, m)) \leq m-1+k$.

Since the "equator" of the sphere $S^{k+1}(s(m))$ contains $N(k, m)$ dispersed points, by adding the two "poles," we see that $N(k+1, m) \geq N(k, m)+2$. And since $N(1, m)=2$, we have

$$
N(k, m) \geq 2 k \text {. }
$$

Hence the next corollary follows.

Corollary $1[6] . \quad \operatorname{sph} K(m, 2 n) \leq m-1+n$.

Let us define

$$
d(m, n)=m-1+\min \{k: N(k, m) \geq n\}
$$

Then Theorem 1 is written as

$$
\operatorname{sph} K(m, n) \leq d(m, n) .
$$

We conjecture that, for $m \leq n$, $\operatorname{sph} K(m, n)=d(m, n)$. In fact, this is true for infinitely many $m, n$ (see Corollary 3 and Theorem 4 below).

Using the results of Schütte and Van der Waerden [13], the values of $d(m, n)$ for $m \leq 4, n \leq 10$, are calculated in [8], which are given in Table 1 .

Theorem 2. For $n \geq 3, \operatorname{sph} K(n, n)>n$.

This and Theorem 1 give

$$
\operatorname{sph} K(4,4)=5
$$

Let $\mathrm{R}(m, n)$ denote the "Ramsey number."

\section{Theorem 3. Suppose that}

$$
m+k \geq \max \{n, \mathrm{R}(m-1, n)\}+1, \quad M \geq \max \{m+k, N(k, m)-n+m+k\} .
$$

Table 1. The values of $d(m, n)$ for $m \leq 4, n \leq 10$.

\begin{tabular}{llllllllll}
\hline & & \multicolumn{8}{c}{$n$} \\
\cline { 2 - 10 } & 2 & 3 & 4 & 5 & 6 & 7 & 8 & 9 & 10 \\
\hline 1 & 1 & 2 & 2 & 2 & 3 & 3 & 3 & 3 & 3 \\
2 & 2 & 3 & 3 & 3 & 4 & 4 & 4 & 5 & 5 \\
3 & 3 & 4 & 4 & 5 & 5 & 5 & 6 & 6 & 6 \\
4 & 4 & 5 & 5 & 6 & 6 & 7 & 7 & 7 & 7 \\
\hline
\end{tabular}


Then $\operatorname{sph} K(m, M) \geq m+k$. For $m=5$, we can replace $\mathrm{R}(m-1, n)$ by $\mathrm{R}(3, n)$ in the condition.

Letting $n=2, M=N(k, m)+m+k-2, k \geq 1$, we have Corollary 2 .

Corollary 2. $\operatorname{sph} K(m, N(k, m)+m+k-2) \geq m+k$ for $k \geq 1$.

For $m \leq 3, k \geq m-1$, then letting $n=m+k-1$ and $M=N(k, m)+1$, the conditions of Theorem 3 are satisfied (because $\mathrm{R}(2, n)=n$, and $N(k, m) \geq 2 k$ ). Hence sph $K(m, N(k, m)+1) \geq m+k$. Combining this result with Theorem 1 , we have the following.

Corollary 3. If $m \leq 3$, then $\operatorname{sph} K(m, n)=d(m, n)$.

Furthermore,

Theorem 4. For any fixed integer $m>1$, $\operatorname{sph} K(m, n)=d(m, n)$ holds for infinitely many $n$.

Since $\mathrm{R}(3,3)=6$ and $N(2, m)=4, N(3, m)=6$ for $m \geq 4$, letting $n=2, m=4$, and $k=2$ in Theorem 3 , we have sph $K(4,8) \geq 6$. Similarly, by letting $n=3$, $m=4, k=3 ; n=3, m=5, k=2$; and $n=3, m=5, k=3$, we have sph $K(4,10) \geq 7$, sph $K(5,8) \geq 7$, and sph $K(5,11) \geq 8$, respectively. Combining these results with the upper bounds obtained from Corollary 1 and Table 1 , we have

$$
6 \leq \operatorname{sph} K(4,8) \leq 7 \leq \operatorname{sph} K(5,8) \leq 8 \leq \operatorname{sph} K(5,11)
$$

and another exact value

$$
\operatorname{sph} K(4,10)=7
$$

If a graph $G$ is isomorphic to a unit neighborhood graph in which any adjacent points are closer than a prescribed distance $\delta<1$, then $G$ is said to be $\delta$ embeddable. Then there arises a question: is there a $\delta<1$ such that every finite simple graph is $\delta$-embeddable? The answer is NO.

Theorem 5. If $0<\delta<1$ and $n>1 /\left(1-\delta^{2}\right)$, then $K(n, n)$ is not $\delta$-embeddable.

\section{Dispersed Set}

In this section we recall some results on dispersed sets and then prove Theorem 5 first. The circumsphere (c-sphere) of a nonempty compact set $X$ in Euclidean $n$-space $E^{n}$ is the sphere of minimum radius that encloses $X$; its radius, denoted by $c(X)$, is called the $c$-radius of $X$; its center is the $c$-center of $X$. If $X$ is the vertex set of a regular $(m-1)$-dimensional simplex of unit side length, then the c-radius of $X, c(X)$, is denoted by $c(m)$. Then it is not difficult to see

$$
c(m)=\sqrt{(m-1) /(2 m)} .
$$


Recall the definition of $s(m)$ :

$$
s(m)=\sqrt{(m+1) /(2 m)} .
$$

Thus we have $c(m)^{2}+s(m)^{2}=1$. Similarly, for any nonempty compact set $X$ with $c(X)<1$, we define $s(X)>0$ by

$$
c(X)^{2}+s(X)^{2}=1 .
$$

The following two theorems are proved in [8].

Theorem A. If $X$ is a dispersed set of size $m$, then $c(X)>c(m)$.

A point set $X$ is said to be c-spherical if all points of $X$ lie on the c-sphere of the set $X$. For example, the vertex set of a regular simplex is c-spherical, but the vertex set of an obtuse triangle is not c-spherical. A point set $X$ is said to be affinely independent if $X$ is the vertex set of nondegenerate simplex.

Theorem B. If $X$ is a dispersed set with $c(X) \leq \sqrt{\frac{1}{2}}$, then $X$ is affinely independent and c-spherical.

Now we proceed to the proof of Theorem 4. First we prepare a lemma. For two nonempty finite sets $X$ and $Y$ in a Euclidean space, we define

$$
\operatorname{dis}(X, Y)=\max \{|x-y|: x \in X, y \in Y\} \text {. }
$$

Lemma 1. $c(X)^{2}+c(Y)^{2} \leq \operatorname{dis}(X, Y)^{2}$.

Proof. We may assume that the c-center of $X$ is at the origin $O$. Let $\left\{x_{1}, \ldots, x_{m}\right\}=$ $\{x \in X:|x|=c(X)\}$. Then the origin $O$ belongs to the convex hull of $\left\{x_{1}, \ldots, x_{m}\right\}$, as is easily seen. Hence $O$ is expressed as a convex combination of $x_{1}, \ldots, x_{m}$ :

$$
O=a_{1} x_{1}+\cdots+a_{m} x_{m} \quad\left(a_{1}+\cdots+a_{m}=1, a_{i} \geq 0\right) .
$$

For any $y$ of $Y$,

$$
\operatorname{dis}(X, Y)^{2} \geq\left|x_{i}-y\right|^{2}=|y|^{2}+c(X)^{2}-2\left\langle y, x_{i}\right\rangle,
$$

where $($,$) denotes the inner product. Multiplying both sides by a_{i}$ and summing on $i$, we have

$$
\operatorname{dis}(X, Y)^{2} \geq|y|^{2}+c(X)^{2}
$$

Hence $\operatorname{dis}(X, Y)^{2} \geq \max _{y}|y|+c(X)^{2} \geq c(X)^{2}+c(Y)^{2}$.

Proof of Theorem 5. Suppose that $K(n, n)$ is $\delta$-embeddable in a Euclidean space. Then there exist two dispersed sets $X, Y$ each of size $n$, such that $\operatorname{dis}(X, Y)<\delta$. By Lemma 1 , we have $c(X)^{2}+c(Y)^{2} \leq \operatorname{dis}(X, Y)^{2}<\delta^{2}$. Hence we may assume $c(X)^{2}<\delta^{2} / 2$. Since $c(X)>c(n)$ by Theorem $A$, we have $c(n)^{2}=$ $(n-1) /(2 n)<\delta^{2} / 2$, i.e., $n<1 /\left(1-\delta^{2}\right)$. Therefore, if $n>1 /\left(1-\delta^{2}\right)$, then $K(n, n)$ is not $\delta$-embeddable. 


\section{Focal Set}

Let $X$ be a nonempty set in $E^{k}$. A point $y$ of $E^{k}$ is called a focal point of $X$ if $|y-x| \leq 1$ for all points $x$ of $X$. The set of all focal points of $X$ is called the focal set of $X$ and it is denoted by $F(X)$. Thus, by definition,

$$
F(X)=\left\{y \in E^{k}:|y-x| \leq 1 \text { for all } x \text { of } X\right\}=\bigcap_{x \in X} B(x, 1),
$$

where $B(x, 1)$ denotes the unit (closed) ball centered at $x \in E^{k}$. Note that $F(X)=\varnothing$ if the c-radius $c(X)$ is $>1$.

The operation $F$ is interesting in its own right. For example, if $X$ is a set of c-radius $\leq 1$, then the set

$$
\frac{1}{2}[F(X)+F(F(X))]
$$

is a set of constant width 1 containing $X$, where + denotes the vector sum of two sets, see [5] for detail.

The following lemma will be clear.

Lemma 2. There exists a dispersed set $X$ of size $m$ in $E^{k}$ such that $\operatorname{dpn} F(X) \geq n$ if and only if $\mathrm{sph} K(m, n) \leq k$.

Example. For two points $x, y(x \neq y)$ in $E^{2}$, the set $F(\{x, y\})$ is the intersection of the two unit disks with centers $x, y$. It is easy to see that if $|x-y|>1$, then dpn $F(\{x, y\}) \leq 2$. Hence $\operatorname{sph} K(2,3)>2$.

By the dimension of a set $X$ in a Euclidean space, we mean the dimension of the flat (=affine subspace) spanned by $X$. In the rest of this section we assume the following:

$X$ is a c-spherical set in $E^{k}$ of dimension $j<k$ with c-center at the origin $O$ and c-radius $c(X)<1$.

The subspace spanned by $X$ is called the tangent space of $X$, and is denoted by $T(X)$. Clearly, $T(X)$ contains the origin $O$ and $\operatorname{dim} T(X)=j$. The orthogonal complement of $T(X)$ in $E^{k}$ is called the normal space of $X$, which is denoted by $N(X)$. Then the intersection of the normal space $N(X)$ and the focal set $F(X)$ is a $(k-j)$-dimensional disk of radius $s(X)=\left(1-c(X)^{2}\right)^{1 / 2}$, because $|x|=$ $c(X)$ for all $x$ of $X$. This disk is called the normal disk and its boundary is called the normal sphere of $X$. For example, if $X=\left\{\left(-\frac{1}{2}, 0,0\right),\left(\frac{1}{2}, 0,0\right)\right\}$ in $E^{3}$, then the normal sphere of $X$ is $\left\{(0, s, t): s^{2}+t^{2}=\frac{3}{4}\right\}$. Note that the normal sphere of $X$ is the intersection of all unit hyperspheres $S(x, 1)$ with center $x \in X$. 
Proof of Theorem 1. Let $X$ be the vertex set of an $(m-1)$-dimensional regular simplex of side-length $1+\varepsilon$ in $E^{m-1+h}$. Then the c-radius of $X$ is $c(X)=$ $(1+\varepsilon) c(m)$, and the normal sphere of $X$ is a $k$-dimensional sphere with radius $s(X)=\left(1-c(X)^{2}\right)^{1 / 2}$. By the definition of $N(k, m)$, a $k$-dimensional sphere of radius $s(m)$ contains a dispersed set of size $N(k, m)$. Hence, if $\varepsilon>0$ is sufficiently small, the normal sphere of $X$ also contains a dispersed set of size $N(k, m)$. Hence dpn $F(X) \geq N(k, m)$. Then by Lemma 2, we have sph $K(m, N(k, m)) \leq$ $m+k-1$.

Let

$$
\begin{aligned}
& p: E^{k} \rightarrow T(X), \\
& q: E^{k} \rightarrow N(X),
\end{aligned}
$$

be the orthogonal projections. Then for any point $z$ of $E^{k}$,

$$
z=p(z)+q(z)
$$

Any noncollinear three points $x, y, z$ determine a circle. By the circular arc $x y z$, we mean the arc $x y z$ of the circle determined by $x, y, z$. The boundary of $F(X)$ is denoted by $\partial F(X)$.

Lemma 3. Let $z$ be a point on the boundary $\partial F(X)$ such that $p(z) \neq 0, q(z) \neq 0$. Let $z^{+}, z^{-}$be the two points where the line $O p(z)$ meets the normal sphere of $X$. Then the circular arc $M(z)=z^{+} z z^{-\cdots}$ lies entirely on $\partial F(X)$.

The $\operatorname{arc} M(z)$ is called the meridian of $\partial F(X)$ passing through the point $z$. Note that $z^{-}=-z^{+}$.

Proof. Since $z$ is a boundary point of $F(X), z$ lies on some sphere $S\left(x_{i}, 1\right)$. And since $z^{+}, z^{-}$are two points of the normal sphere of $X, z^{+}$and $z^{-}$lie on $S\left(x_{j}, 1\right)$ for all $j=1, \ldots, m$. Here we note the following fact: if a circle and a sphere have more than two points in common, then the circle lies entirely on the sphere. Therefore, if $z \in S\left(x_{j}, 1\right)$, then $M(z) \subset S\left(x_{j}, 1\right)$, while if $z \notin S\left(x_{j}, 1\right)$, then, since $z \in B\left(x_{j}, 1\right)$, all points of $M(z)$ other than the two endpoints are interior points of $B\left(x_{j}, 1\right)$. Therefore $M(z) \subset F(X)$ and $M(z) \subset S\left(x_{i}, 1\right)$. Thus $M(z) \subset \partial F(X)$.

\section{Dispersed Points in a Focal Set}

In this section we prove some lemmas that are useful in the computation of the dispersed point number of a focal set. Throughout this section, we assume that $X=\left\{x_{1}, \ldots, x_{m}\right\} \subset E^{k}(m \leq k)$ is a dispersed set with c-center $O$ (origin) and c-radius $c(X) \leq \sqrt{\frac{1}{2}}$. 
Then by Theorem B, $X$ is affinely independent and c-spherical; the tangent space $T(X)$ of $X$ is an $(m-1)$-dimensional subspace of $E^{k}$, and the normal space $N(X)$ (the orthogonal complement of $T(X)$ in $\left.E^{k}\right)$ is $(k-m+1)$-dimensional. The projections $E^{k} \rightarrow T(X), E^{k} \rightarrow N(X)$ are denoted by $p$ and $q$, respectively. Further, we denote by

$$
f: E^{k}-\{O\} \rightarrow \partial F(X)
$$

the central projection from the origin, i.e., for any $z \neq O, f(z)$ is the point where the ray $\overrightarrow{O z}$ meets the boundary $\partial F(X)$. (Note that $F(X)$ is a convex body containing $O$ inside.)

Lemma 4. For any dispersed pair $x, y$ in $F(X)$,

$$
y \neq O \text { and }|x-f(y)| \geq|x-y| .
$$

Proof. Since $|x|<1$ (because $\left|x-x_{i}\right| \leq 1$ for $i=1, \ldots, m$, and $O$ is contained in the convex hull of $X$ ) and $|y|<1$, it follows that $x \neq O, y \neq O$, and that the angle at $O$ is the largest angle in the triangle $O x y$. Then the angle $\angle O y x$ is acute, and hence it follows that $|x-f(y)| \geq|x-y|$.

Let us denote by $\Delta(X)$ the simplex spanned by $X$.

Lemma 5. Let $z$ be a point of $F(X)$. If $|z| \geq c(X)$, then $p(z) \in \Delta(X)$.

Proof. We prove the contraposition. Suppose that $z^{\prime}=p(z)$ lies outside $\Delta(X)$. Then, for some $0<t<1, t z^{\prime}$ lies on a face of $\Delta(X)$, say on the face opposite to the point $x_{1}$. Then $t z^{\prime}$ is expressed as a convex combination of $x_{2}, \ldots, x_{m}$ :

$$
t z^{\prime}=a_{2} x_{2}+\cdots+a_{m} x_{m}
$$

Since $1<\left|x_{1}-x_{i}\right|^{2}=2 c(X)^{2}-2\left\langle x_{1}, x_{i}\right\rangle$ for $i \geq 2$, we have $2\left\langle x_{1}, x_{1}\right\rangle<2 c(X)^{2}-1$, and

$$
\begin{aligned}
2\left\langle x_{1}, t z^{\prime}\right\rangle & =2\left\langle x_{1}, a_{2} x_{2}+\cdots+a_{m} x_{m}\right\rangle \\
& <\left(a_{2}+\cdots+a_{m}\right)\left(2 c(X)^{2}-1\right)=2 c(X)^{2}-1<0 .
\end{aligned}
$$

So $2\left\langle x_{1}, z^{\prime}\right\rangle\left\langle 2 c(X)^{2}-1\right.$. Since $\left\langle x_{1}, z\right\rangle=\left\langle x_{1}, z^{\prime}\right\rangle$,

$$
1 \geq\left|x_{1}-z\right|^{2}=c(X)^{2}+|z|^{2}-2\left\langle x_{1}, z\right\rangle>c(X)^{2}+|z|^{2}-2 c(X)^{2}+1 .
$$

Therefore $|z|<c(X)$.

Lemma 6. $\quad c(F(X) \cap T(X))<c(X)$.

Proof. First note that $x_{i} \notin F(X), i=1, \ldots, m$, for $X$ is dispersed. Let $z \in F(X) \cap$ $T(X)$. Then $z=p(z)$. If $z \in \Delta(X)$, then since $z \neq x_{i}, i=1, \ldots, m,|z|<c(X)$. If $z \notin \Delta(X)$, then, by Lemma $5,|z|<c(X)$. 
For a point $z$ of $F(X)$, let us define

$$
z^{\circ}=\left\{\begin{array}{lll}
f(p(z)) & \text { if } & p(z) \neq O, \\
O & \text { if } & p(z)=O,
\end{array} \quad z^{+}=\left\{\begin{array}{lll}
f(q(z)) & \text { if } & q(z) \neq O \\
O & \text { if } & q(z)=O .
\end{array}\right.\right.
$$

If $p(z) \neq O$ and $q(z) \neq O$, then the circular $\operatorname{arc} z^{+} z^{\circ}\left(-z^{+}\right)$is the meridian $M(f(z))$ passing through the point $f(z)$ (cf. Lemma 3 ). In this case, $z^{\circ}$ bisects the meridian $M(f(z))$, and $z$ lies on the angular region $\angle z^{\circ} O z^{+}$. Note also that if $z \neq O$, then $z^{+}=f(z)^{+}$and $z^{\circ}=f(z)^{\circ}$.

Lemma 7. Let $x, y$ be a dispersed pair on $\partial F(X)$ such that $\langle x, q(y)\rangle \geq O$. If $|y|<c(X)$, then $\left|x-y^{\circ}\right| \geq|x-y|$, while if $|y| \geq c(X)$, then $\left|x-y^{+}\right| \geq|x-y|$.

Proof. If $p(y)=O$, then $|y|=\left|y^{+}\right|=s(X)>c(X)$, and $\left|x-y^{+}\right|=|x-y|$. If $q(y)=$ $O$, then $|y|=\left|y^{\circ}\right|<c(X)$, and $\left|x-y^{\circ}\right|=|x-y|$. Assume $p(y) \neq O$ and $q(y) \neq O$. Let $M(y)$ be the meridian of $\partial F(X)$ passing through $y$, and let $P$ be the plane determined by $M(y)$ (i.e., $P$ is determined by $O, p(y), q(y)$ ). Let $w$ be the center of the circle $C$ determined by $M(y)$. Then since the circle $C$ passes through $y^{+}$ and $y^{-}:=-y^{+}$, the center $w$ must lie on the line $O y^{\circ}$. Since $\left|y^{\circ}\right|<c(X)<s(X)=$ $\left|y^{+}\right|$, the origin lies between $w$ and $y^{\circ}$, and hence there is a unique point $z$ on the subarc $y^{+} y^{\circ}$ of $M(y)$ such that $|z|=c(X)$. The point $y$ lies on the $\operatorname{arc} y^{+} z$ or on the arc $z y^{\circ}$ of $M(y)$ accordingly as $|y| \geq c(X)$ or $|y|<c(X)$. Let $x^{\prime}$ be the projection of the point $x$ on the plane $P$ determined by $C$.

Suppose first $|y|<c(X)$. Since $\langle x, q(y)\rangle \geq 0$, the points $x^{\prime}$ and $y^{+}$lie on the same side of the line $O y^{\circ}$ in the plane $P$. In this case one of the angles $\angle x^{\prime} w z$, $\angle x^{\prime} w y^{\circ}$ is greater than $\angle x^{\prime} w y$. And hence one of $\left|x^{\prime}-z\right|,\left|x^{\prime}-y^{\circ}\right|$ is greater than $\left|x^{\prime}-y\right|$, from which we can deduce that one of $|x-z|,\left|x-y^{\circ}\right|$ is greater than $|x-y|$. Thus if we prove $|x-z| \leq 1$, then we have $\left|x-y^{\circ}\right| \geq|x-y|$. Since $|z|=c(X)$, $p(z)$ is contained in $\Delta(X)$ by Lemma 5 . Hence $p(z)$ is expressed as a convex combination of $x_{1}, \ldots, x_{m}$ :

$$
p(z)=a_{1} x_{1}+\cdots+a_{m} x_{m} \quad\left(a_{1}+\cdots+a_{m}=1, a_{i} \geq 0\right) .
$$

For each $x_{i}$,

$$
1 \geq\left|x-x_{i}\right|^{2}=|x|^{2}+\left|x_{i}\right|^{2}-2\left\langle x, x_{i}\right\rangle
$$

Multiplying both sides by $a_{i}$ and summing on $i=1, \ldots, m$, we have

$$
1 \geq|x|^{2}+c(X)^{2}-2\langle x, p(z)\rangle=|x|^{2}+|z|^{2}-2\langle x, p(z)\rangle .
$$

But since $\langle x, z\rangle=\langle x, p(z)\rangle+\langle x, q(z)\rangle$, we have

$$
|x-z|^{2}=|x|^{2}+|z|^{2}-2\langle x, p(z)\rangle-2\langle x, q(z)\rangle \leq 1-2\langle x, q(z)\rangle \leq 1 .
$$

Thus $|x-z| \leq 1$.

Similarly, if $|y| \geq c(X)$, then we have $\left|x-y^{+}\right| \geq|x-y|$. 
Lemma 8. Let $x, y$ be a dispersed pair in $F(X)$. Then $\left|x^{+}-y^{+}\right|>1$ or $\left|x^{\circ}-y^{\circ}\right|>1$ holds. If $|y| \geq c(X)$, then $\left|x^{+}-y^{+}\right|$is always greater than 1 .

Proof. We may assume that $x, y$ lie on the boundary $\partial F(X)$ (otherwise, by Lemma 4 , we can replace $x, y$ by $f(x), f(y))$. If $\langle x, q(y)\rangle<0$, then $\left\langle x^{+}, y^{+}\right\rangle<0$, and hence $\left|x^{+}-y^{+}\right|^{2}>\left|x^{+}\right|^{2}+\left|y^{+}\right|^{2}=2 s(X)^{2}>1$. Suppose now $\langle x, q(y)\rangle \geq 0$. Then, by the above lemma, $\left|x-y^{+}\right|>1$ or $\left|x-y^{\circ}\right|>1$ accordingly as $|y| \geq c(X)$ or $|y|<c(X)$. Since $\left\langle y^{\circ}, q(x)\right\rangle=0$ and $\left\langle y^{+}, q(x)\right\rangle \geq 0$, applying Lemma 7 again, and noting that $\left|x^{\circ}-y^{+}\right|,\left|x^{+}-y^{\circ}\right| \leq 1$, we have $\left|x^{\circ}-y^{\circ}\right|>1$ or $\left|x^{+}-y^{+}\right|>1$. If $|y| \geq c(X)$, then $\left|x^{+}-y^{+}\right|$is always greater than 1 .

\section{Proof of Theorems 2-4}

Proof of Theorem 2. Suppose that $\operatorname{sph} K(n, n) \leq n$ for some $n \geq 3$. Then there exist two dispersed sets $X, Y$, each of size $n$ in $E^{n} \operatorname{such}$ that $\operatorname{dis}(X, Y) \leq 1$. We may assume $c(X) \leq c(Y)$ and the c-center of $X$ is at the origin $O$. Then, by Lemma $1, c(X) \leq \sqrt{\frac{1}{2}}$, and hence the tangent space $T(X)$ of $X$ is $(n-1)$ dimensional by Theorem B. Hence the normal space of $X$ is one-dimensional, and the normal sphere of $X$ consists of only two points. Since $c(X) \leq c(Y)$, there is at least one point $y$ in $Y$ such that $|y| \geq c(X)$. Then, since $Y$ is a dispersed set in $F(X)$, applying Lemma 8 , we have $\left|y^{+}-w^{+}\right|>1$ for all $w$ of $Y-\{y\}$. Hence, all points $w^{+}$must coincide with each other. Then, again by Lemma 8 , we must have $\left|v^{\circ}-w^{\circ}\right|>1$ for $v, w \in Y-\{y\}$. This implies that $X$ and $\left\{w^{\circ}: w \in Y, w \neq y\right\}$ together induce a unit neighborhood graph isomorphic to $K(n, n-1)$, and hence sph $K(n, n-1) \leq n-1$. Thus $\operatorname{sph} K(n-1, n-1) \leq n-1$. Repeating the same argument, we finally reach $\operatorname{sph} K(2,3) \leq 2$, which is a contradiction (see the example after Lemma 2).

Proof of Theorem 3. Suppose that $\operatorname{sph} K(m, M) \leq m-1+k$. Then there exist two dispersed sets $X, Y$ of size $m$ and $M$ in $E^{m-1+k}$ such that $F(X) \supset Y$. If $c(Y)<c(X)$, then $c(Y)<\sqrt{\frac{1}{2}}$ by Lemma 1 , and $Y$ is affinely independent and c-spherical by Theorem $B$. In this case, $Y$ spans $E^{m-1+k}$, and since $X \subset F(Y)$, we must have $c(X)<c(F(Y))<c(Y)$ by Lemma 6 , which is a contradiction. Hence $c(X) \leq c(Y)$. Therefore $X$ is affinely independent and c-spherical. We may assume that the c-center of $X$ is at the origin $O$. Let

$$
W=\{w \in Y:|w|<c(X)\} .
$$

Then $|W| \leq m+k$. However, $|W|=m+k$ is impossible, because if $|W|=m+k$, then $W$ spans $E^{m-1+k}$, and since $X \subset F(W)$, we must have $c(X)<c(W)<c(X)$, a contradiction. Thus $|W| \leq m-1+k$. Therefore, there are at least

$$
N(k, m)-n+1
$$

points $y_{i}, i=1, \ldots, N(k, m)-n+1$, in $Y$ such that $\left|y_{i}\right| \geq c(X)$. Let

$$
U=Y-\left\{y_{i}: i=1, \ldots, N(k, m)-n+1\right\} .
$$


Let $G^{\circ}$ be the graph with vertex set $U$ and edge set

$$
\left\{u v: u, v \in U \text { and }\left|u^{\circ}-v^{\circ}\right| \leq 1\right\}
$$

Then since $|U| \geq m-1+k \geq \max \{n, R(m-1, n)\}$ and since $n=R(2, n)$, the graph $G^{\circ}$ contains either an independent set of size $\max \{2, m-1\}$ or a clique of size $n$. We show that $G^{\circ}$ must contain a clique of size $n$. If $m \leq 3$, then since

$$
\operatorname{sph} K(1,2)=1, \quad \operatorname{sph} K(2,2)=2, \quad \text { and } \quad \operatorname{sph} K(3,2)>2 \text {, }
$$

$F(X) \cap T(X)$ cannot contain two dispersed points, and hence $G^{\circ}$ is a complete graph. Suppose that for $m>3, G^{\circ}$ contains an independent set of size $m-1$. Then the set $\left\{u^{\circ}: u \in U\right\}$ contains $m-1$ dispersed points, and hence $T(X)$ contains a unit neighborhood graph isomorphic to $K(m, m-1)$. But since

$$
\operatorname{sph} K(m-1, m)>m-1
$$

by Theorem 2 , this is impossible. Thus, in either case, $G^{\circ}$ must contain a clique of size $n$. Let $\left\{u_{i}: i=1, \ldots, n\right\}$ be a clique of $G^{\circ}$. Then, by Lemma 8 , the set

$$
\left\{u_{i}^{+}: i=1, \ldots, n\right\} \cup\left\{y_{1}^{+}: i=1, \ldots, N(k, m)-n+1\right\}
$$

is a dispersed set of size $N(k, m)+1$ on the normal sphere of $X$. However, since the normal sphere of $X$ is a $k$-dimensional sphere of radius $s(X)<s(m)$, it cannot contain more than $N(k, m)$ dispersed points. Thus we have a contradiction. Therefore, $\operatorname{sph} K(m, M) \geq m+k$.

Now assume $m=5$. By Corollary 3 , and Table 1 , we have sph $K(3,5)=5$ (note that to derive Corollary 3 , we used only the case $m \leq 3$ of Theorem 3 ). Hence we have $\mathrm{dpn}(F(X) \cap T(X)) \leq 2$. Therefore we may replace $\mathrm{R}(m-1, n)$ by $\mathrm{R}(3, n)$ in the condition of the theorem

Proof of Theorem 4. We proved in Theorem 3 of [3] that, for any fixed $m>0$, $N(k, m)$ is exponentially large in $k$, that is,

$$
N(k, m)>(k-2) /(k-1)^{1 / 2} \exp \left\{(k-2) \beta^{2} / 2\right\}
$$

where $\beta=\sin ^{-1}\{1 /(m+1)\}$. Hence, for infinitely many $k, N(k+1, m)-$ $N(k, m)>k+m$ holds (for otherwise, $N(k, m)<k^{2} / 2+O(k)$, which is not exponentially large). Then, by Corollary 2 and Theorem 1 ,

$$
m+k \leq \operatorname{sph} K(m, N(k, m)+m+k-2) \leq \operatorname{sph} K(m, N(k+1, m)) \leq m+k
$$

holds for infinitely many $k$. For each of such $k$, let $n=n(k)=N(k+1, m)$. Then $d(m, n)=m+k$ and $\operatorname{sph} K(m, n)=d(m, n)$. 


\section{References}

1. P. C. Fishburn, On the sphericity and cubicity of graphs, J. Combin. Theory Ser. B 35 (1983), 309-318.

2. P. Frankl and H. Maehara, Embedding the $n$-cube in lower dimensions, European J. Combin. 7 (1986), 221-225.

3. P. Frankl and H. Maehara, On the contact dimension of graphs, Discrete Comput. Geom. 3 (1988), 89-96.

4. L. C. Freeman, Spheres, cubes, and boxes: graph dimensionality and network structure, Social Network 5 (1983), 139-156.

5. H. Maehara, Convex bodies forming pairs of constant width, J. Geom. 22 (1984), 101-107.

6. H. Maehara, Space graphs and sphericity, Discrete Appl. Math. 7 (1984), 55-64.

7. H. Maehara, On the sphericity for the join of many graphs, Discrete Math. 49 (1984), 311-313.

8. H. Maehara, Contact patterns of equal nonoverlapping spheres, Graphs Combin. 1 (1985), 271-282.

9. H. Maehara, Sphericity exceeds cubicity for almost all complete bipartite graphs, J. Combin. Theory Ser. B 40 (1986), 231-235.

10. H. Maehara, On the sphericity of the graphs of semi-regular polyhedra, Discrete Math. 58 (1986), 311-315.

11. H. Maehara, J. Reiterman, V. Rödl, and E. Šimňajova, Embedding trees in Euclidean spaces, Graphs Combin. 4 (1988), 43-47.

12. J. Reiterman, V. Rödl, and E. Šiňajova, Geometrical embedding of graphs, Discrete Math., to appear.

13. K. Schutte and B. L. Van der Waerden, Auf welcher kugel haben 5, 6, 7, 8, order 9 punkte mit Mindestabstand Ein Platz?, Math. Ann. 123 (1951), 96-124.

Received December 17, 1987, and in revised form September 7, 1988. 